\title{
SYT-SSX fusion is absent in sarcomatoid mesothelioma allowing its distinction from synovial sarcoma of the pleura
}

\author{
Nicolas Weinbreck ${ }^{1}$, Jean Michel Vignaud ${ }^{1,2}$, Hugues Begueret ${ }^{2}$, Louise Burke ${ }^{3}$, \\ Jean Benhattar ${ }^{4}$, Louis Guillou ${ }^{2,4}$, Frédérique Capron ${ }^{2}$ and Françoise Galateau-Salle ${ }^{2,5}$ \\ ${ }^{1}$ Departement de Pathologie, CHU de Nancy and EMI 014, Nancy, France; ${ }^{2}$ Groupe Mesopath, ERI 3 Inserm, \\ CHU Caen, Caen, France; ${ }^{3}$ Cork University Hospital, Cork, Ireland; ${ }^{4}$ University Institute of Pathology, \\ Lausanne, Switzerland and ${ }^{5}$ ERI 3 Inserm, CHU Caen, Caen, France
}

\begin{abstract}
The diagnosis of sarcomatoid mesothelioma is still a worldwide challenge and it is often difficult, both clinically and by morphological analysis, to differentiate sarcomatoid mesothelioma from synovial sarcoma, the most frequent intrathoracic sarcoma. To confirm the absence of the synovial sarcoma translocation $t(X ; 18)(S Y T$ SSX) in sarcomatoid mesothelioma, and to test its usefulness differentiating sarcomatoid mesothelioma from synovial sarcoma, 28 tumours were examined using the reverse transcriptase-polymerase chain reaction. RNA was extracted from paraffin blocks using standard methods, reverse-transcribed and PCR performed. Molecular analysis completed in two independent laboratories showed that sarcomatoid mesothelioma samples were negative for the $\mathrm{t}(\mathrm{X}-18)$. This result confirms the usefulness of this analysis in differentiating sarcomatoid mesothelioma from synovial sarcoma.
\end{abstract}

Modern Pathology (2007) 20, 617-621; doi:10.1038/modpathol.3800775

Keywords: sarcomatoid mesothelioma; RT-PCR; t(X; 18); SYT-SSX; synovial sarcoma

The sarcomatoid variant of malignant mesothelioma accounts for about $11 \%^{1}$ of all pleural mesotheliomas. The diagnosis of sarcomatoid mesothelioma is still a challenge, with recognisable pitfalls in its distinction from organising fibrous pleuritis, sarcomatoid carcinomas, primary and metastatic sarcomas. Distinguishing sarcomatoid mesothelioma from synovial sarcoma can be extremely difficult, and although synovial sarcoma is the most frequent primitive intrathoracic sarcoma, its rarity in the pleural location makes it an easily overlooked differential. The importance of making the correct diagnosis is further highlighted by the fact that synovial sarcoma is usually chemosensitive ${ }^{2}$ while sarcomatoid mesothelioma is chemoresistant. ${ }^{3,4}$ Classic biphasic synovial sarcoma is usually easy to recognize by light microscopy; however, most of the intrathoracic synovial sarcoma cases are monophasic histological subtypes with a predominance of monophasic fibrous cases creating the diagnostic challenge (Figure 1). As these tumours share the

Correspondence: Dr JM Vignaud, MD, Departement de Pathologie, Hôpital Central, CHU de Nancy, 54035 Nancy Cedex, France.

E-mail: jm.vignaud@chu-nancy.fr

Received 2 January 2007; revised 15 February 2007; accepted 16 February 2007 same clinical presentation and as immunostains do not reliably differentiate between a sarcomatoid mesothelioma and monophasic spindle cell or poorly differentiated synovial sarcoma, additional tests are required. Both tumours typically express keratin, may express calretinin and are negative for glycoprotein markers. The advent of molecular techniques has increased both sensitivity and specificity of the diagnosis of synovial sarcoma by detecting the specific $t(X ; 18)$ translocation. ${ }^{5}$ The demonstration of the translocation $t(X ; 18)$ in paraffin-embedded material using reverse transcription-polymerase chain reaction (RT-PCR) is now considered as a reliable and powerful tool for the diagnosis of synovial sarcoma owing to the high sensitivity and specificity of this method. The translocation results in the formation of a chimeric gene that encodes a transcription-activating protein. ${ }^{6}$ This translocation leading to the fusion of $S Y T$ (at 18q11) with either SSX1 or SSX2 (two closely related genes at Xp11), or rarely $S S X 4$ (also at Xp11), represents the most specific and sensitive tool for diagnosing a synovial sarcoma. ${ }^{5,7,8}$ Bégueret et $a l^{9}$ confirmed the presence of this translocation in $90 \%$ of purely sarcomatoid primary synovial sarcoma of the pleura. To verify the absence of the synovial sarcoma translocation in sarcomatoid mesothelioma, 

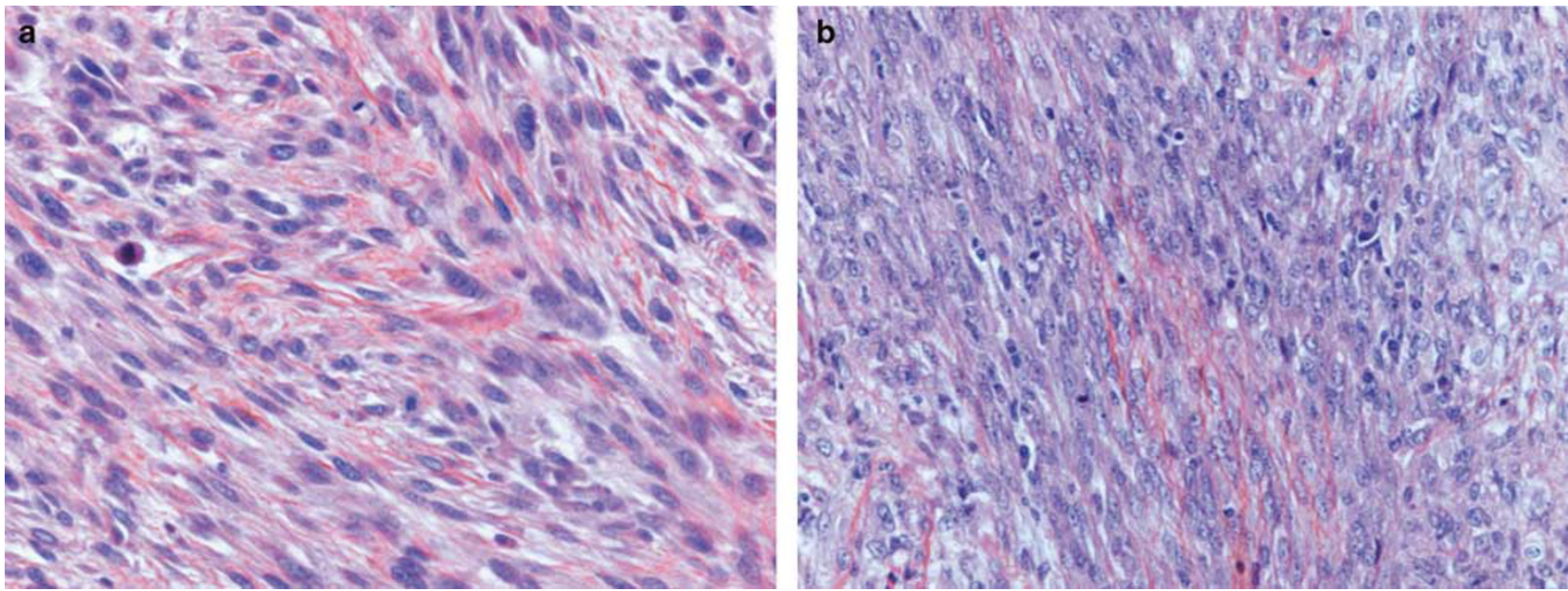

Figure 1 Pleural biopsies from patients a and $\mathbf{b}$, presenting with diffuse pleural tumour, displayed very similar H\&E pattern and immunohistochemical profile. Only the presence of $S Y T-S S X$ gene transcripts in sample tissue from patient a allowed to reliably identity the tumour as monophasic synovial sarcoma, whereas because of the absence from patient b's tumour it was diagnosed as sarcomatoid mesothelioma.

28 tumour samples were examined, in two independent laboratories, using RT-PCR. In none of our cases of sarcomatoid mesothelioma was SYT-SSX fusion transcript detected.

\section{Materials and methods}

All sarcomatoid mesothelioma cases included in this study were validated according to the procedure of certification previously defined. ${ }^{1}$ Briefly, each case included in the French National Mesothelioma Surveillance Program (PNSM) undergoes a standardized diagnostic confirmation procedure by a National group of pathologists who specialize in mesothelioma, the National Mesopath group. Three experts, blinded to asbestos exposure, classify each case as certain, uncertain, unclassifiable because of inadequate materials, or rule out in favour of another diagnosis, on the base of haematoxylin and eosin sections and immunohistochemical documents. When all three experts reach the same diagnosis, it is considered confirmed. Otherwise, the case is reviewed collectively in consensus meetings involving at least 10 experts and confirmed, ruled out, or referred for clinical expertise.

For this study 32 cases of sarcomatoid mesothelioma were identified from the files of the Mesopath group. There were two women and 30 men. All had a history of asbestos exposure and a highly suggestive clinical and CT-scan presentation for sarcomatoid mesothelioma. Three intrathoracic synovial sarcomas bearing the $\mathrm{t}(\mathrm{X} ; 18)$, used as positive control, were retrieved from the files of the Department of Pathology of Nancy University Hospital (France). All tissue samples had been fixed in $4 \%$ phosphate buffered-formalin. Histological typing was performed on haematoxylin and eosin sections using the WHO histological classification of lung and pleural tumours. ${ }^{10}$
The most frequently utilized monoclonal and polyclonal antibodies for immunohistochemical studies included: Calretinin (polyclonal, human recombinant Zymed, San Francisco, CA, USA), Cytokeratin 5/6 (clone D5/16B4, Dakocytomation, Glostrup, Denmark), cytokeratin AE1-AE3 (Dakocytomation), cytokeratin KL1 (Immunotech, Marseille, France), multicytokeratin (clone C11, Novocastra Laboratories Ltd, Newcastle upon Tyne, UK), epithelial membrane antigen (clone E29, Dakocytomation), CD34 (clone QBEND 10, Immunotech), smooth muscle actin alpha (clone alpha sm-1, Novocastra), desmin (clone D33, Dakocytomation), S100 protein (Dakocytomation), CD99 (clone M3601, Dakocytomation), bcl-2 (clone bcl-2/100/D5, Novocastra). Immunostaining was performed applying the biotin streptavidin method. Furthermore samples stained with multicytokeratin antibodies (AE1-AE3, KL1 and C11) and calretinin, additionally used a tyramin-biotin signal amplification procedure (Catalyzed Signal Amplification System, DakoCytomation), according to the manufacturer's instructions. Antigen retrieval was processed in citrate buffer ( $\mathrm{pH} \mathrm{6)}$ ), using a pressure cooker. All steps were performed at room temperature, and diaminobenzidine was used as a chromogen. Appropriate positive and negative controls were used throughout.

\section{RNA Extraction}

Approximately $50 \mathrm{mg}$ of tumour were removed from paraffin blocks. The tissue samples were deparaffinized by incubation for $5 \mathrm{~min}$ in $1 \mathrm{ml}$ xylene at $65^{\circ} \mathrm{C}$; this reaction was repeated to remove residual paraffin. After centrifugation, the tissue was washed with $1 \mathrm{ml}$ of $100 \%$ ethanol, followed by $1 \mathrm{ml}$ of $70 \%$ ethanol. After $5 \mathrm{~min}$ of vacuum drying, the pellet was suspended in $250 \mu \mathrm{l}$ of lysis buffer $(20 \mathrm{mmol} / \mathrm{l}$ Tris-HCl, $\mathrm{pH} 7.5 ; 20 \mathrm{mmol} / \mathrm{l}$ ethylenediaminetetra- 
acetic acid; $1 \%$ sodium dodecyl sulfate; $350-500 \mu \mathrm{g}$ proteinase $\mathrm{K}$ ). After 16 to $48 \mathrm{~h}$ incubation at $55^{\circ} \mathrm{C}$, $750 \mu \mathrm{l}$ of Trizol-LS reagent (Gibco BRL, Gainthersburg, MD, USA) was added to the sample, and total RNA was extracted according to the manufacturer's instructions. The RNA pellet was dissolved in $40 \mu \mathrm{l}$ of RNase-free water and stored at $-80^{\circ} \mathrm{C}$. The quantity of the isolated RNA was determined by absorbance at $260 \mathrm{~nm}$ and $280 \mathrm{~nm}$.

\section{RT-PCR Analysis}

All samples were analysed in two independent laboratories using the same techniques (Institute of Pathology, Lausanne, Switzerland and Département de Pathologie, Nancy, France).

The RT-PCR amplification was performed with the Superscript One-Step RT-PCR System (Invitrogen, Carlsbad, CA, USA) in a final volume of $20 \mu \mathrm{l}$ containing 100-200 ng of total RNA, with 10 pM of each primer ( $2 \mathrm{pM}$ of each $\beta$-actin primer). Reverse transcription was done at $50^{\circ} \mathrm{C}$ for $30 \mathrm{~min}$. After inactivation of the reverse transcriptase at $95^{\circ} \mathrm{C}$ for $5 \mathrm{~min}, 40$ cycles of PCR amplification were performed at $95^{\circ} \mathrm{C}$ for $30 \mathrm{~s}, 57^{\circ} \mathrm{C}$ for $45 \mathrm{~s}$, and $72^{\circ} \mathrm{C}$ for $75 \mathrm{~s}$. To check the quality of every mRNA sample, we always co-amplified a ubiquitously expressed control gene ( $\beta$-actin). The primers were: SYT-A (5'-CAG CAGAGGCCTTATGGATATGA-3'), $S S X$-B (5'-TTTGT GGGCCAGATGCTTC- $\left.3^{\prime}\right), \beta$-actin-A (5'-AGGCCAAC CGCGAGAAGATGA-3 ${ }^{\prime}$ ) and $\beta$-actin-B (5'-GCCGTG GTGGTGAAGCTGTAG-3'). The reaction products were subjected to electrophoresis in $2 \%$ agarose gel and visualized by ethidium bromide staining. The size of PCR products was $97 \mathrm{bp}$ for the SYT-SSX (SYT$S S X 1$ or $S Y T-S S X 2)$ fusion gene transcripts and $274 \mathrm{bp}$ for the $\beta$-actin messenger RNA, which was used as an internal positive control. The RT-PCR procedure was performed at least twice for each sample.

For each PCR procedure, a classic $t(X ; 18)$-positive biphasic synovial sarcoma was used as a positive control. Negative controls consisted of $t(X ; 18)$ negative normal lung parenchyma, as well as distilled water devoid of template cDNA.

\section{Results}

\section{Immunohistochemical Findings in the Sarcomatoid Mesothelioma Cases}

All sarcomatoid mesothelioma tumour samples were variably positive for cytokeratin markers, either KL1 or AE1-AE3 (two cocktails containing antibodies to both high and low molecular-weight cytokeratins), with areas of strong staining admixed with areas of absent staining. It was also noted that, in $30 \%$ of cases, a reciprocal staining intensity pattern was observed-ie cases with strong staining for AE1-AE3 displayed weak staining for KL1 or the reverse profile. Using standard biotin streptavidin amplification procedures, 15 out of 32 samples

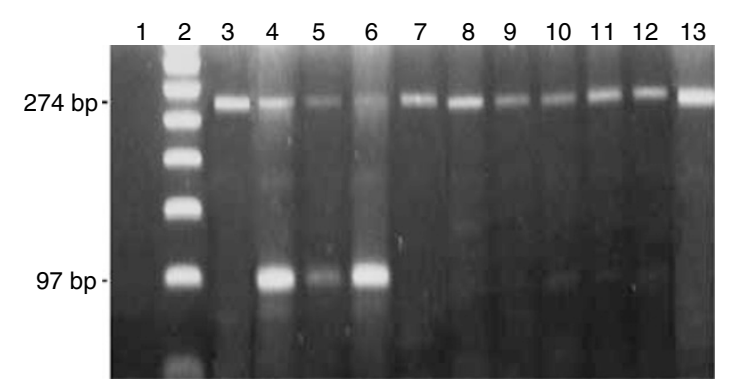

Figure 2 RT-PCR shows the absence of SYT-SSX transcripts in sarcomatoid mesotheliomas. Lane 1 (distilled water devoid of template cDNA) and 3 (normal lung parenchyma) are negative controls; lanes 4, 5, 6, synovial sarcoma positive controls and lanes 7 to 13 sarcomatoid mesotheliomas. PCR products are $97 \mathrm{bp}$ for the SYT-SSX (SYT-SSX1 or SYT-SSX2) fusion gene transcripts and $274 \mathrm{bp}$ for the $\beta$-actin messenger RNA. Lanes 4 and 11 correspond respectively to tumours samples from patients a and $b$ presented in Figure 1.

(54\%) showed nuclear staining for calretinin, rarely diffuse $(28 \%)$, mostly focal. Tyramin-biotin signal amplification showed a clear cut increase in the percentage of calretinin expressing cells, but none of the tumour samples negative for calretinin using standard biotin streptavidin amplification procedures were subsequently stained positively. Thirtyfour percent of tumour samples stained positively for CK5/6, 28\% for EMA, 20\% for CD34 and 22\% for Bcl2. All tumour samples were negative for smooth muscle actin, muscle-specific actin, desmin, CD99 and S100.

\section{Molecular Genetic Findings}

Interpretable results were obtained from 28 cases only $(87.5 \%)$. All 28 sarcomatoid mesotheliomas were negative for the $\mathrm{t}(\mathrm{X} ; 18)$ (SYT-SSX translocation) in the presence of positive PCR internal controls ( $\beta$-actin) (Figure 2). Four tumour samples from four patients (out of 32) showed negative results for both $\beta$-actin and SYT-SSX fusion gene transcripts and thus were not included in the analysis. Three classic synovial sarcoma cases used as positive controls showed the $\mathrm{t}(\mathrm{X} ; 18)$ translocation.

\section{Discussion}

Sarcomatoid mesotheliomas account for about $11 \%{ }^{1}$ of all pleural mesotheliomas, and typically consist of spindle cells arranged haphazardly or in fascicles. Their differential diagnosis includes benign and malignant processes such as organising fibrous pleuritis, sarcomatoid carcinomas, primary and metastatic sarcomas and more specifically synovial sarcoma. ${ }^{11}$ Pleural metastases are a frequent event in the clinical course of soft tissue sarcomas, but primary intrathoracic sarcomas are rare. However, synovial sarcoma probably account as the most common of all primary intrathoracic sarcomas. ${ }^{12}$ Examining a series of 40 intrathoracic synovial sarcomas, Bégueret et $a l^{9}$ found that $27 \%$ involved 
the pleura and $77.5 \%$ the pleura and lung. $97.5 \%$ of intrathoracic synovial sarcoma cases were of the monophasic histologic subtype, $60 \%$ being monophasic fibrous and $37.5 \%$ poorly differentiated with a high histologic grade, but epithelial differentiation was nevertheless detectable in $90 \%$ of them in the form of focal EMA and/or keratin reactivity. Because of its rarity and microscopic presentation in this location synovial sarcoma can be easily misdiagnosed as another type of tumour especially with sarcomatoid mesothelioma and their diagnosis most often requires molecular analysis (RT-PCR). Immunohistochemical staining is insufficient to definitively distinguish between these two malignancies. Indeed sarcomatoid mesothelioma and monophasic spindle cell or poorly differentiated synovial sarcoma show an overlap in their respective immunohistochemical spectrum with a variable staining for EMA, low and high molecular-weight keratin, calretinin, vimentin, S-100 protein and Bcl2, and with CD34 negativity. To date such immunohistochemical evaluation is not reliable in making the distinction between sarcomatoid mesothelioma and synovial sarcoma, especially when dealing with small specimens or poorly differentiated tumours. ${ }^{11}$

Additionally, clinical findings do not significantly help in differentiating the two entities as similar symptoms including pleural effusion, chest pain, cough, and haemothorax occur in both. Although the median age of patients presenting with synovial sarcoma is about 48 years vs 69 years for mesothelioma in Begueret's series, ${ }^{9} 10 \%$ of patients with intrathoracic synovial sarcoma were over 70 years, thus making young age a non-reliable discriminant feature in individual cases.

At the time of diagnosis most of the cases of synovial sarcoma $(80 \%)$ presented as circumscribed masses and truly localized mesotheliomas are quite uncommon $;{ }^{13}$ however in $20 \%$ of cases both entities share a similar presentation. ${ }^{9}$ Occupational exposure to asbestos does not definitively rule out the diagnosis of synovial sarcoma, as in France $65 \%$ of men over 65 years have a history of occupational exposure to asbestos. ${ }^{14}$ As a consequence the true incidence of synovial sarcoma of the pleura is unknown, because only in the recent past has progress in molecular medicine provided the tools to reliably identify this tumour. However, there is growing evidence that synovial sarcoma may develop as a primary tumour in the pleura. ${ }^{15-20}$ In this regard during the last five years (2001-2005), 3209 malignant mesotheliomas were certified by the French Mesothelioma panel (Mesopath group), 353 were sarcomatoid mesotheliomas (11\%). Among sarcomatoid mesotheliomas, 54 were poorly differentiated, pleomorphic tumours $(1.7 \%$ of all malignant mesotheliomas, $14 \%$ of sarcomatoid mesotheliomas). During the same period of time 58 primitive synovial sarcomas involving the pleura, all but one monophasic and all bearing the $t(X ; 18)$, considered as sarcomatoid mesotheliomas by the pathologists responsible for the initial diagnosis, were addressed to the Mesopath group (about 2 cases of synovial sarcoma for 100 certified malignant mesotheliomas). Given the distinct strategies for clinical management of these tumour types, it is essential that pleural synovial sarcomas are not misdiagnosed as sarcomatoid mesotheliomas or sarcomatoid carcinomas. Although more aggressive than their soft tissue counterparts synovial sarcomas are less aggressive than sarcomatoid mesotheliomas with a 50 months median survival for synovial sarcoma ${ }^{9}$ vs 9 months for sarcomatoid mesothelioma (Mesopath group data). As already detailed, clinical findings, histology and immunohistochemical features are insufficient to definitively distinguish between these two malignancies, detection of the $\mathrm{t}(\mathrm{X} ; 18)$ translocation using RT-PCR is considered as the most powerful test to make this distinction. Despite past contradictory reports, ${ }^{21-23}$ the presence of $S Y T-S S X$ fusion transcripts is now considered as a diagnostic signature of synovial sarcoma. ${ }^{24-27}$ Guillou et $a 2^{24}$ reported a prospective study of 211 mesenchymal tumours fixed in different fixatives, in which there were 135 non-synovial sarcoma tumours, 22 biphasic and 64 monophasic spindle/round cell synovial sarcomas. SYT-SSX gene fusion transcripts were detected in the synovial sarcoma tumour category only ( $100 \%$ specificity) with an overall sensitivity of $96 \%$. This sensitivity level is in keeping with results reported in other series by Hiraga $(100 \%)^{26}$ and Bijwaard (96.5\%). ${ }^{27}$ Malignant mesothelioma karyotypic analysis studies performed during the past several decades failed to identify a specific karyotypic change that may prove to be of diagnostic value and showed the complexity of the karyotypes in this malignancy. Most malignant mesotheliomas display multiple clonal chromosomal abnormalities. ${ }^{28-29}$ However a number of recurrent anomalies have been found. These include deletions at multiple sites or loss of one entire copy for chromosomes 4 and 22, polysomy for chromosomes $5,7,20$, and losses at 1p21-2, and 3p21, 6q15-21, 9p21-22, 15q11.1-q15 and 22q12. Comparative genomic hybridisation studies confirmed the karyotype findings and identified even more chromosomal losses and gains than either conventional cytogenetics or FISH. ${ }^{30}$ None of the significant genetic changes reported concern chromosome X or 18.

Thus because of its rarity and microscopic presentation in the thorax, synovial sarcoma can be easily misdiagnosed as another type of tumour especially with sarcomatoid mesothelioma. Taking in consideration the importance of differentiating sarcomatoid mesothelioma from synovial sarcoma in terms of treatment, survival and medico legal compensation, we looked for the $\mathrm{t}(\mathrm{X}$; 18) translocation in a series of optimally validated sarcomatoid mesotheliomas. All our samples were processed in duplicate in two independent laboratories with similar results obtained in both, contributing to demonstrate the specificity, sensibility and reprodu- 
cibility of RT-PCR technnique. Therefore as none of the sarcomatoid mesotheliomas displayed the $\mathrm{t}(\mathrm{X} ; 18)$, in the face of appropriate positive and negative controls, we can state that absence of the translocation when dealing with this differential strongly supports the diagnosis of sarcomatoid mesothelioma.

\section{Acknowledgement}

We thank Lydia Brochin for excellent technical assistance.

\section{References}

1 Goldberg M, Imbernon E, Rolland P, et al. The French National Mesothelioma Surveillance Program. Occup Environ Med 2006;63:390-395.

2 Testa JR, Pass HI, Carbone M. Molecular Biology of Mesothelioma. In: Devita VT, Hellman S, Rosenberg SA (eds). Cancer Principles and Practice of Oncology, 6th edn. PA Lippincott Williams and Wilkins: Philadelphia, 2001, pp 1937-1943.

3 Weiss SW, Goldblum JR. Soft Tissue Tumors, 4th edn. MI Mosby Inc.: St Louis, 2001, pp 199-245.

4 Pass HI, Robinson BW, Testa JR, et al. Emerging translational therapies for mesothelioma. Chest 1999;116:455S-460S.

5 Coindre JM, Pelmus M, Hostein I, et al. Should molecular testing be required for diagnosing synovial sarcoma? A prospective study of 204 cases. Cancer 2003;98:2700-2707.

6 Clark J, Rocques PJ, Crew AJ, et al. Identification of novel genes, $S Y T$ and $S S X$, involved in the $\mathrm{t}(\mathrm{X} ; 18)$ (p11.2;q11.2) translocation found in human synovial sarcoma. Nat Genet 1994;7:502-508.

7 Hisaoka M, Hashimoto H, Iwamasa T, et al. Primary synovial sarcoma of the lung: report of two cases confirmed by molecular detection of SYT-SSX fusion gene transcripts. Histopathology 1999;34: 205-210.

8 Pfeifer JD, Hill DA, O’Sullivan MJ, et al. Diagnostic gold standard for soft tissue tumours: morphology or molecular genetics? Histopathology 2000;37: $485-500$.

9 Bégueret H, Galateau-Salle F, Guillou L, et al. Primary intrathoracic synovial sarcoma: a clinicopathologic study of $40 \mathrm{t}(\mathrm{X} ; 18)$ - positive cases from the french sarcoma group and the mesopath group. Am J Surg Pathol 2005;29:339-346.

10 Travis WD, Brambilla E, Muller-Hermelink HK, et al. (eds). World Health Organization Classification of Tumors. Tumors of the Lung, Pleura, Thymus and Heart. IARC Press: Lyon, 2004.

11 Galateau-Salle F, Brambilla E, Cagle P, et al. In: Galateau-Sallé (ed). Pathology of Malignant Mesothelioma; an Update of the International Mesothelioma Panel. Springer Verlag: New York, 2006.

12 Keel SB, Bacha E, Mark EJ, et al. Primary pulmonary sarcoma: a clinicopathologic study of 26 cases. Mod Pathol 1999;12:1124-1131.

13 Allen TC, Cagle PT, Churg AM, et al. Localized malignant mesothelioma. Am J Surg Pathol 2005; 29:866-873.
14 Rapport d'activité 1998-2002 PNSM. DST-Institut de Veille Sanitaire. Hopital Saint Maurice: France.

15 Aubry MC, Bridge JA, Wickert R, et al. Primary monophasic synovial sarcoma of the pleura: five cases confirmed by the presence of SYT-SSX fusion transcript. Am J Surg Pathol 2001;25:776-781.

16 Essary LR, Vargas SO, Fletcher CD. Primary pleuropulmonary synovial sarcoma: reappraisal of a recently described anatomic subset. Cancer 2002;94: 459-469.

17 Gaertner E, Zeren EH, Fleming MV, et al. Biphasic synovial sarcomas arising in the pleural cavity. A clinicopathologic study of five cases. Am J Surg Pathol 1996;20:36-45.

18 Jawahar DA, Vuletin JC, Gorecki P, et al. Primary biphasic synovial sarcoma of the pleura. Respir Med 1997;91:568-570.

19 Nicholson AG, Goldstraw P, Fisher C. Synovial sarcoma of the pleura and its differentiation from other primary pleural tumours: a clinicopathological and immunohistochemical review of three cases. Histopathology 1998;33:508-513.

20 Roggli V. Pleural lesions. Histopathology 2002;41: 147-177.

21 O’Sullivan MJ, Kyriakos M, Zhu X, et al. Malignant peripheral nerve sheath tumors with $t(X$;18). A pathologic and molecular genetic study. Mod Pathol 2000;13:1253-1263.

22 Ladanyi M, Woodruff JM, Scheithauer BW, et al. Letter to the editor. Mod Pathol 2001;14:733-737.

23 Coindre JM, Hostein I, Benhattar J, et al. Malignant peripheral nerve sheath tumors are $\mathrm{t}(\mathrm{X}-18)$-negative sarcomas. Molecular analysis of 25 cases occurring in neurofibromatosis type 1 patient, using two different RT-PCR-based methods of detection. Mod Pathol 2002;15:589-592.

24 Guillou L, Coindre JM, Gallagher G, et al. Detection of the synovial sarcoma translocation $t(X ; 18)$ $(S Y T ; S S X)$ in paraffin-embedded tissue using reverse transcriptase-polymerase chain reaction: a reliable and powerful diagnostic tool for pathologists. A molecular analysis of 221 mesenchymal tumors fixed in different fixatives. Hum Pathol 2001;32: 105-112.

25 Fukuoka K. Molecular detection of SYT-SSX fusion gene transcripts currently represents the most specific and sensitive tool for diagnosing intrathoracic synovial sarcoma. Intern Med 2006;45:881-882.

26 Hiraga H, Nojima T, Abe S, et al. Diagnosis of synovial sarcoma with the reverse transcriptase-polymerase chain reaction: analyses of 84 soft tissue and bone tumors. Diagn Mol Pathol 1998;7:102-110.

27 Bijwaard KE, Fetsch JF, Prygodzki R, et al. Detection of $S Y T-S S X$ fusion transcripts in archival synovial sarcomas by real-time reverse transcriptase-polymerase chain reaction. J Mol Diagn 2002;4:59-64.

28 Sandberg AA, Bridge JA. Updates on the cytogenetics and molecular genetics of bone and soft tissue tumors. Mesothelioma. Cancer Genet Cytogenet 2001;127:93-110.

29 Taguchi T, Jhanwar SC, Siegfried JM, et al. Recurrent deletions of specific chromosomal sites in 1p, 3p, 6q, and $9 p$ in human malignant mesothelioma. Cancer Res 1993;53:4349-4355.

30 Lee WC, Testa JR. Somatic genetic alterations in human malignant mesothelioma. Int $\mathrm{J}$ Oncol 1999;14:181-188. 